\title{
DIE RECHTSVERFOLGUNG
}

IM

\section{INTERNATIONALEN VERKEHR.}

\section{DARSTELLUYG}

DER JUSTIZORGANISATION, DES PROZESSRECHTS, DES KONKURSRECHTS UND DER ERBSCHAFTSREGULIRUNG IN DEN KULTURSTAATEN DES ERDBALLS.

UNTER

MITWIRKCYG HERVORRAGENDER JURISTEN ALLER LÄNDER HERAUSGEGEBEN

voN

Dr. W. REULING, und Dr. W. LOEWENFELD, RECHTSANWALT BEIM REICHSGERICHT ZU JEIPZIG, RECHTSANWALT BEIM LANDGERICIT I ZU RERI.IN.

\section{ERSTE LIEFERUNG.}

(DEUTSCHES REICH, OESTERREICH-UNGARN EINSCHLIESSLICH BOSNIEN UND HERZEGOWINA, SCHWEIZ, NIEDERLANDE.)

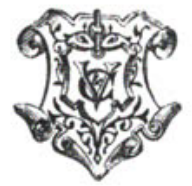

LEIPZIG, VERLAG VON VEIT \& COMP. 1887. 